\title{
Posterior First and Second Cervical Vertebrae Fusion by Screw Fixation Technique using the Modern Pre-fabricated Template Method on Cadaver Samples
}

\author{
Athari $\mathbf{M}^{1}$, MD, Golbakhsh $\mathbf{M R}^{2}$, MD, Mirbolook $\mathbf{A}^{1}$, MD, Athari $\mathbf{M}^{1}$, MD, Ahmadi $\mathbf{A}^{2}, \mathrm{MD}$, \\ Komlakh K $\mathbf{K}^{3}$, MD, Azarhomayoun $\mathbf{A}^{4}$, MD, Paydarniya $\mathbf{P}^{5}$, MD \\ 'Department of Orthopaedics, Shahid Beheshti University of Medical Sciences, Tehran, Iran \\ ${ }^{2}$ Department of Orthopaedics, Tehran University of Medical Sciences, Tehran, Iran \\ ${ }^{3}$ Department of Neurosurgery, Shahid Beheshti University of Medical Sciences, Tehran, Iran \\ ${ }^{4}$ Department of Neurosurgery, Sina Trauma and Surgery Research Center, Tehran, Iran \\ ${ }^{5}$ Department of Neurosurgery, Tehran University of Medical Sciences, Tehran, Iran

This is an open-access article distributed under the terms of the Creative Commons Attribution License, which permits unrestricted use,
distribution, and reproduction in any medium, provided the original work is properly cited
Date of submission: 03rd January 2021
Date of acceptance: 29 th July 2021

\begin{abstract}
Introduction: $\mathrm{C} 1$ lateral mass and $\mathrm{C} 2$ pedicular screws insertion are used for $\mathrm{C} 1-\mathrm{C} 2$ posterior fusion. Fluoroscopy Guided technique is routinely used for screw placement but it is associated with risk of injury to spinal cord and vertebral artery. 3D printing has developed rapidly in the fields of medicine. It is helpful in improving precise treatment and used for instrumentation in spine. We want to evaluate the accuracy of $\mathrm{C} 1$ lateral mass screws and $\mathrm{C} 2$ pedicle screws insertion by Pre-Fabricated Template made by threedimensional (3D) printing.

Materials and methods: Five cervical samples were obtained from cadavers. Based on fine-cut CT scan 3Dimages reconstructed and the path of the screws designed by special software. A template produced by 3D-printer from 3D images. After printing the templates, they were fixed on the relevant vertebra in the operation room and based on the template path, $\mathrm{C} 1$ lateral mass screw and $\mathrm{C} 2$ pedicular screws were inserted. Placement of the screws was evaluated using CT scans post-operatively.

Results: A total of 14 screws were inserted by abovementioned method. After evaluation with CT scans none of the screws were entered in the spinal canal. Two screws had vertebral artery canal perforation with less than $50 \%$ breach. Violation was judged as noncritical and would probably not have resulted in injury to vertebral artery.

Conclusions: The accuracy of $\mathrm{C} 1$ lateral mass screw and $\mathrm{C} 2$ pedicle screw insertion is acceptable with pre-fabricated template and can provide a useful aid for screw placement.
\end{abstract}

Keywords:

atlas, axis, three-dimensional, fusion, pre-fabricated template

\section{INTRODUCTION}

The first and second cervical vertebrae are among the most important and complex components of the vertebral column. The anatomy of these two vertebrae and their joints is one of the most complex and sensitive parts of the human body as they are connected by multiple ligaments and joints ${ }^{1,2,3}$. Similar to the other parts of the human body, this structure is prone to a number of diseases and injuries. A wide range of diseases, including inflammatory diseases, e.g., rheumatoid arthritis, congenital conditions, and trauma, can damage this structure leading to atlantoaxial instability ${ }^{1-4}$. The surgical treatment of atlantoaxial instability is performed by fixing the first and second vertebrae using a number of different methods. Among these methods, fixing with screws has been considered in recent years. The surgical treatment of this area is associated with high risk, even if it is performed by the most experienced surgeons, due to the complexity of the anatomy and the sensitivity of the elements in this area. Also, there is several anatomic variations in the general population.

The 3D printing has been employed in many surgical and medical fields. Such may include surgical planning, printing of models to aid with intra-operative guidance and anatomy definition, prosthesis development, medical education, patient-specific implant creation, and numerous other purposes $^{5}$.

Due to the sensitivity of the atlantoaxial area and the complexity of the anatomy, it seems that pre-fabricated templates, which can guide the correct screw fixation path according to each person's anatomy, can reduce the risk of surgery and its adverse consequences. The main objective of

Corresponding Author: MirBahador Athari, Department of Orthopaedics, Shahid Beheshti University of Medical Sciences, Tehran Province, Tehran, Velenjak، 7th Floor, Bldg No.2 SBUMS, Arabi Ave، Iran

Email: Bahadorathari@gmail.com 
this study is to investigate the screw fixation technique for a $\mathrm{C} 1$ lateral mass screw and a pedicle screw in the second vertebra through pre-fabricated templates on cadaver samples.

\section{MATERIALS AND METHODS}

At first, five cervical samples were obtained from the Anatomy Department of the University of Tehran. After numbering the samples, CT scan was performed with a delicate cut of $2 \mathrm{~mm}(130 \mathrm{kv}, 2 \mathrm{mAs})$ at Sina Hospital Imaging Centre. The CT scan data were transferred to the three-dimensioning mimics software [Materialize Mimic software version 19] in the form of DICOM files for preparing Three-Dimensioning CT Scans. In this software, the CT scans taken from the samples are reconstructed in three-dimensional form. The software environment is shown in (Fig. 1).

Then, in the same environment, the correct direction of the screws was determined using cylinders in the software environment. The path for each cylinder was followed in all cuts of axial, coronal and sagittal planes of the CT scan, where each path of the cylinder was in its correct path. None of the cylinders had entered the canal or towards the holes of the vertebral artery (Fig. 2).

With regards to the first and second cervical vertebra, the path of the screw was determined for each sample and examined in various cuts in terms of correctness of the path. Finally, the path of the screws in the three-dimensional view was examined (Fig. 3,4,5).

After designing the path of the screws, the information was stored as an STL file and transferred to the Geomatics Studio program. Then, based on the outer cortex and the posterior cortex of the first and second vertebrae, special templates were designed. These templates are perfectly fixed on the posterior section of each vertebra so that the path of the screws passes through the pre-fabricated templates (Fig. 6). Once the design stage was complete, the samples were prepared with the help of Jarah Yar Sharif Company (Centre for Science and Technology in Medicine, Imam Khomeini Hospital, Tehran, Iran) by printing them in three-dimensional form.

After printing the templates, they were fixed on the relevant vertebra in the operation room at Sina Hospital. Then, based on the template path, a hole was created using a 2.7 drill. Next, a $3.5 \mathrm{~mm}$ screw (Osweh company, Tehran, Iran) was fixed in the drilled position (Fig. 7).

After fixing the screws, the sites of the screws were checked by $\mathrm{CT}$ scan. In the performed $\mathrm{CT}$ scans, samples were examined in terms of extending into the spinal canal and the vertebral artery canal by Kaneyama et al classification ${ }^{6}$. The grades are as follows: Grade 1: None of the canals were damaged. Grade 2: Less than $50 \%$ of the screw is inside the canal. Grade 3: More than $50 \%$ of the screw is inside the canal.

\section{RESULTS}

Four cervical samples were obtained from the anatomy department. Then, four screws were fixed in C1 and C2 of each sample according to the method described. Due to damage to the posterior arch of the cervical vertebra in one of the samples, it was impossible to place the screw in the first vertebra of this sample. Finally, 14 screws were fixed using the abovementioned method. Based on the postoperative CT scan (Fig. 8). the screws were ranked in three grades based on the extent of damage to the spinal canal and the vertebral arterial canal using Kaneyama et al classification for screw ${ }^{6}$.

In this study, none of the screws entered the spinal canal. Two screws were entered in vertebral artery canal that were grade 2 , while none of the screws were in grade 3.

\section{DISCUSSION}

The fixation of a screw in the lateral masses of the first vertebra and the fixation of a screw inside the second vertebra's pedicles require high skill and knowledge about the anatomy of this area. Surgery around theses area is difficult and dangerous due to the relatively small vertebral pedicle and the key anatomical structures around them. Therefore, comprehensive evaluation and precise navigation are very important for the surgery. Even with high levels of experience, there is a risk of damage to the spinal cord and the vertebral artery. The normal navigation methods face the problem of intra-operative drift; thus, the screw may easily break through the bone cortex when the pedicle is small ${ }^{7-11}$. Patient-specific drill guide templates have been developed as an inexpensive, accurate method to guide screw insertion in the spine based on advances in $3 \mathrm{D}$ computing and printing technologies. Ryken et al fixed screws on four cadaver samples for the first time using pre-fabricated templates ${ }^{12}$.

Moreover, D'urso et al showed that the specific template for each vertebra had high accuracy in screw fixation ${ }^{13}$. In addition, Van Cleynenbreugel et al used two types of templates for screw fixation using the Magerl method ${ }^{14}$. Hu et al used a pre-fabricated template for screw fixation using the Magerl method ${ }^{15}$. Sugawara et al used template for posterior $\mathrm{C} 1-\mathrm{C} 2$ fusion. they inserted a total of 48 screws with accuracy of $100 \%{ }^{16}$. Guo et al compared C1 and C2 screw fixation in patients guided by the template and the patients which screws were fixed under guidance of fluoroscopy. In the template guided group, the acceptability 


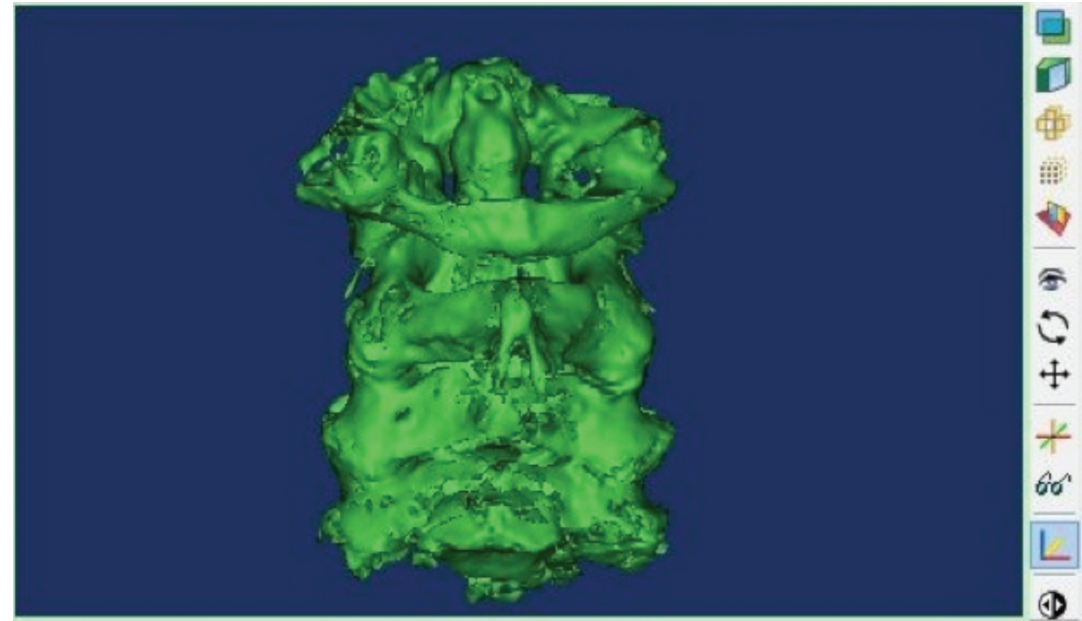

Fig. 1: Medical Mimics Software Environment. This software is able to detect bone tissues according to the assumptions of the software, and it can perform a three-dimensional reconstruction.

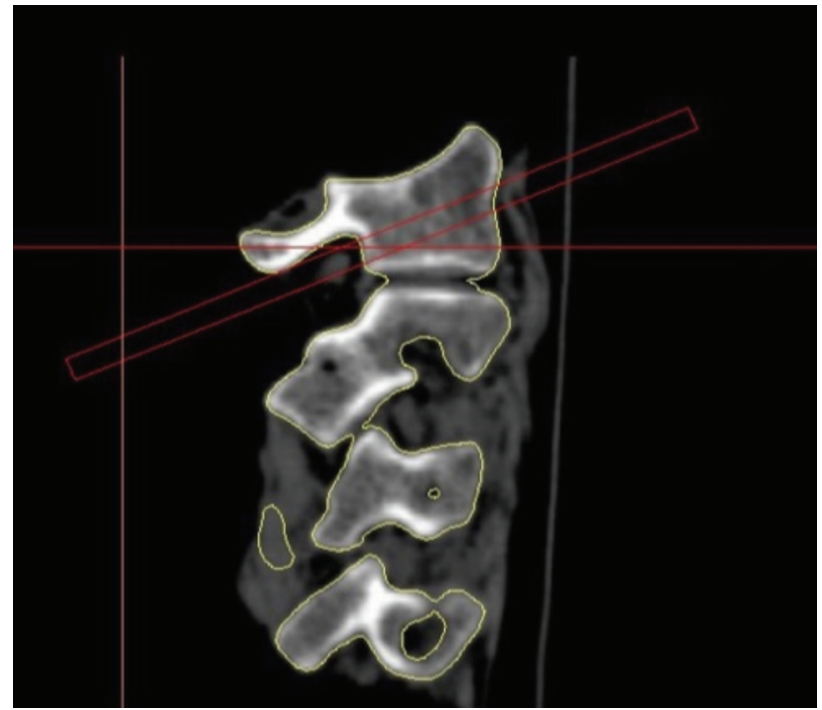

Fig. 2: Sagittal CT from first vertebrae used for surgical planning.

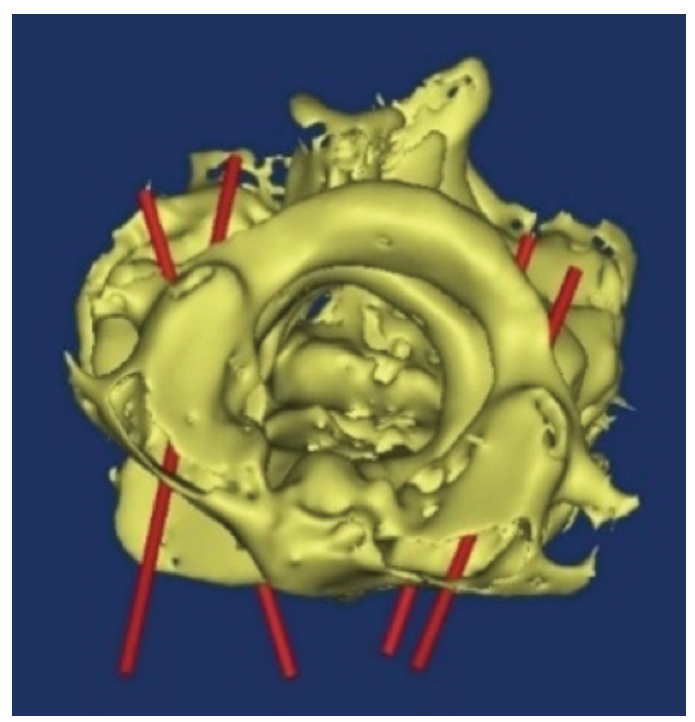

Fig. 4: Three-dimensional axial view. Red cylinders represent the path of the screws.

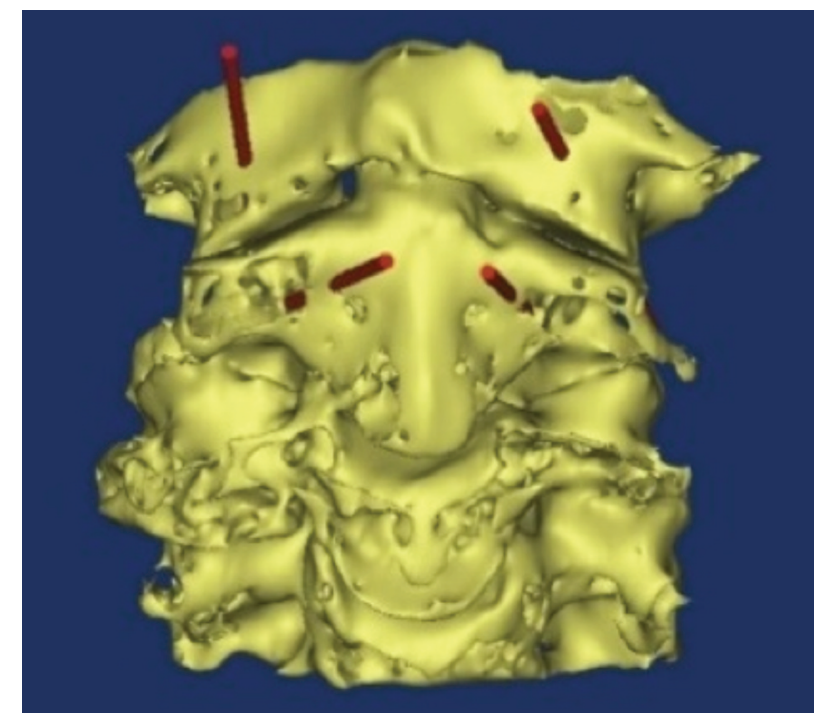

Fig. 3: Three-dimensional coronal view. Red cylinders represent the path of the screws.

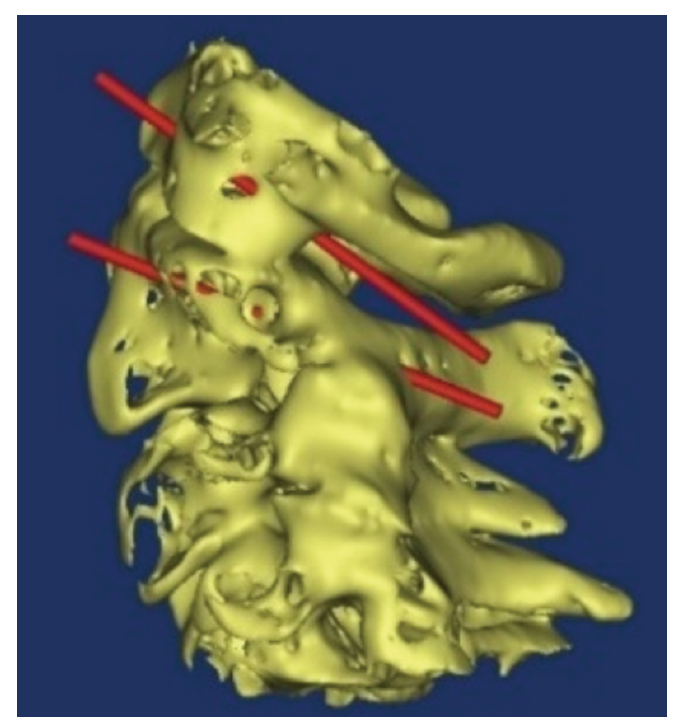

Fig. 5: Three-dimensional axial view. Red cylinders represent the path of the screws. 


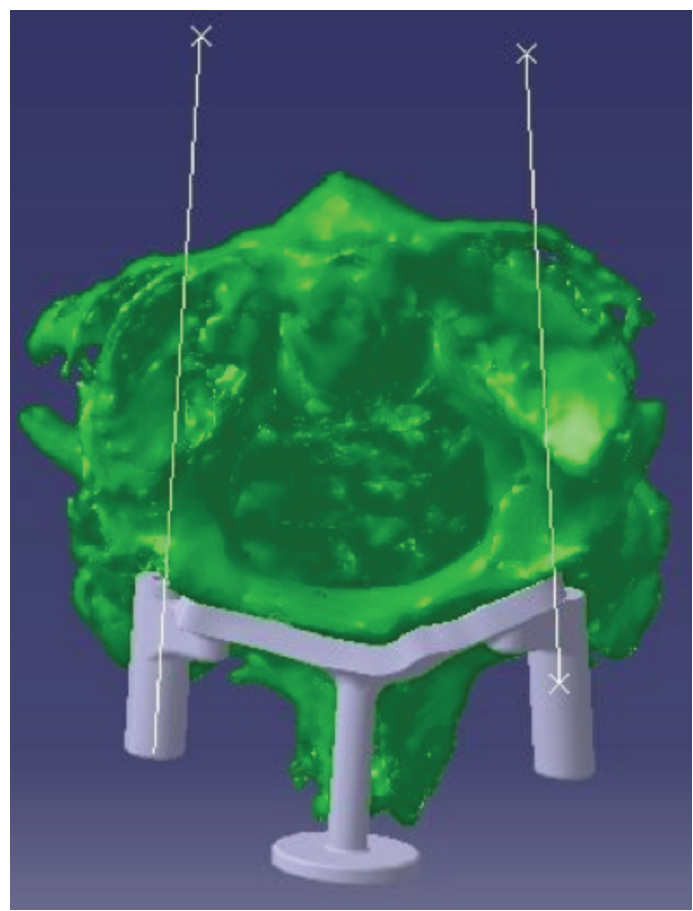

Fig. 6: Templates designed based on the path of the screws. The lines represent the pre-specified path.
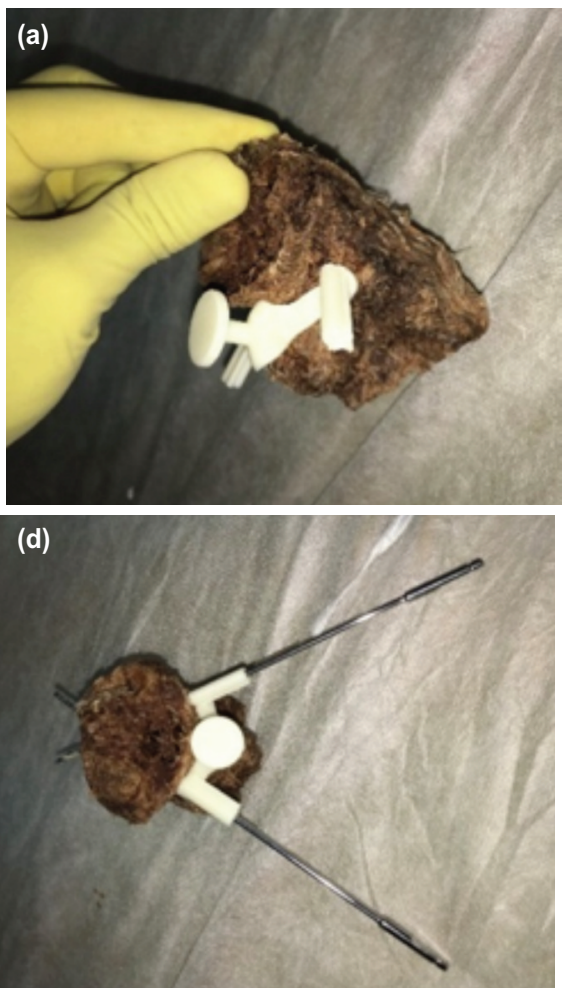

(b)

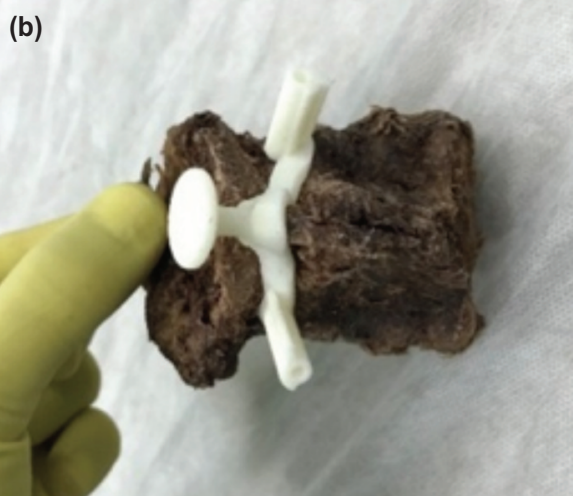

\section{(e)}

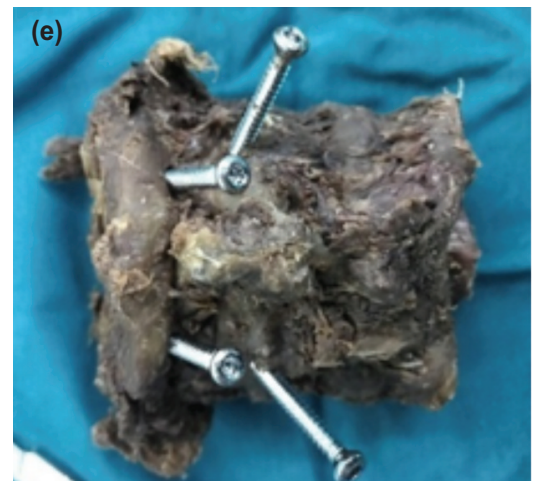

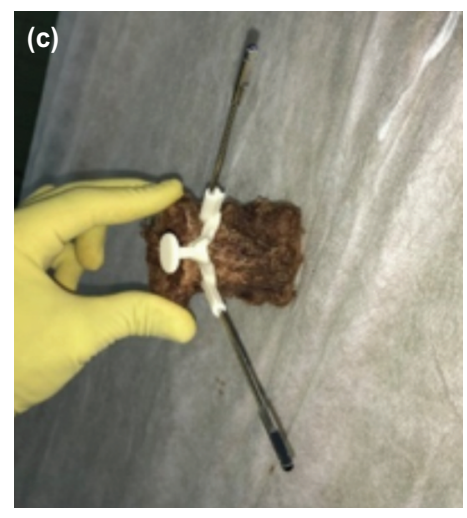

(f)

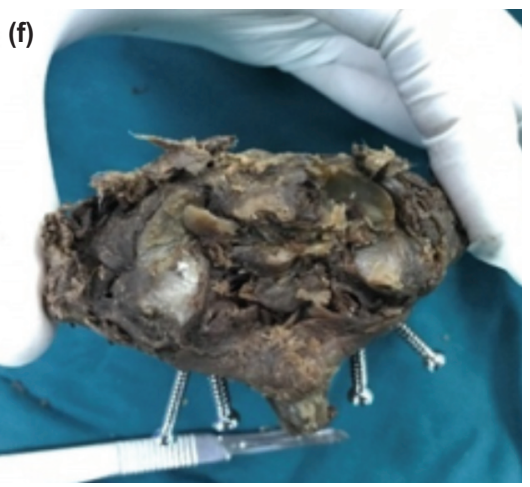

Fig. 7: Stages of screw fixation and a screw- fixed sample. (a) The template fixed over the C2 vertebrae. (b) The template shown on the sample from different angle. (c) The path drilled in the path. (d) Same sample from different angle. (e) The screws inserted after drilling. (f) Same sample shown with the fixed screws. 


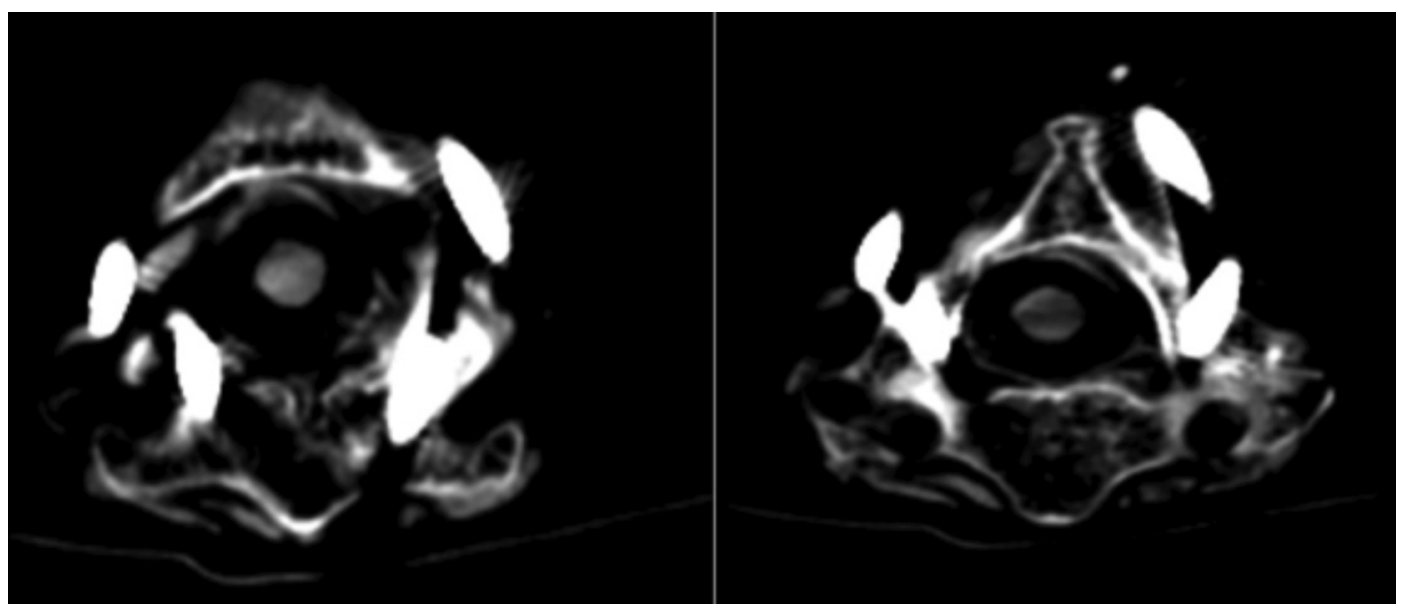

Fig. 8: Post-operative CT scan for checking the screws' site.

rate was $94.6 \%$ compared to $70.27 \%$ in control group. The acceptability rate in test group was higher than the rate in control group. The operation time and fluoroscopic frequency for each screw were decreased, compared with the control group ${ }^{17}$.

In another study comparing a total of 68 screws placed at the pedicle in the 19 patients who underwent atlantoaxial pedicle screw assisted by a 3D-printed guide plate, while a total of 76 screws were placed at the pedicle in 24 patients using the traditional method. The accuracy of screw placement in the 3D-printed plate group (94.1\%) was far higher than that in the traditional fixation group $(76.3 \%)$. atlantoaxial pedicle screw fixation assisted by a 3D-printed plate significantly improved the accuracy rate of screw placement ${ }^{18}$. The navigation template used in the current study was built by several reverse engineering software packages; thus, it required manual adjustments. These factors affect the accuracy of the method, and different studies report different navigation accuracy rates (i.e., 84-97.6\%) (19-22 $^{\text {. }}$

Pre-fabricated samples based on the patient's CT scans try to reduce the error during the surgery and minimise adverse complications of these operations. The screw fixation technique based on pre-fabricated templates is a modern technique, which reduces the risk of such operations. The results of the current study are comparable to the findings of the previous studies. However, the screws damaged the cortex of the vertebral canal in two cases, which may have been due to the very small diameter of the samples' pedicle. Therefore, the screw fixation method is not recommended if the diameter of the pedicle is small.

To our knowledge, this is the first study in Iran for assessment of feasibility and accuracy of a 3D-printed template for spinal instrumentation. The first limitation of this study is the low number of cadaver samples. Unfortunately, access to cadaver samples has many problems in our country and samples often do not have good quality. The soft tissue of these cadaver samples does not have good consolidation. In addition, samples undergo changes in bone density due to long-term storage, which are evident in the CT scans and differ from living samples. Another limitation of this study is that common screws were used, due to the high cost of cervical screws, and these common screws cause artifacts in the post-operative CT scans.

As such, we expect to summarise the advantages and disadvantages of the 3D printing technology in a follow-up work in vivo and promote the relevant clinical applications.

\section{CONCLUSION}

Cervical pedicle screw placement using a rapid prototyping drill template based on a 3D model is technically feasible, and it can enable accurate pedicle screw placement without the need for extra radiation. This study indicates the ability to perform such techniques in Iran; however, further studies are needed in this area.

\section{ACKNOWLEDGEMENT}

We would like appreciate Dr. Sobhani, head of the Anatomy department, for his assistance in providing the samples.

\section{CONFLICT OF INTEREST}

The authors declare no potential of conflict of interest. 


\section{REFERENCES}

1. Tomasino A, Parikh K, Koller H, Zink W, Tsiouris AJ, Steinberger J, et al. The vertebral artery and the cervical pedicle: morphometric analysis of a critical neighborhood. J Neurosurg Spine. 2010; 13(1): 52-60. doi: 10.3171/2010.3.SPINE09231

2. Bydon M, Mathios D, Macki M, De la Garza-Ramos R, Aygun N, Sciubba DM, et al. Accuracy of C2 pedicle screw placement using the anatomic freehand technique. Clin Neurol Neurosurg. 2014; 125: 24-7. doi: 10.1016/j.clineuro.2014.07.017

3. Goel AL, Laheri V. Plate and screw fixation for atlanto-axial subluxation. Acta Neurochir (Wien). 1994; 129(1-2): 47-53. doi: 10.1007/BF01400872

4. Hojo Y, Ito M, Suda K, Oda I, Yoshimoto H, Abumi K. A multicenter study on accuracy and complications of freehand placement of cervical pedicle screws under lateral fluoroscopy in different pathological conditions: CT-based evaluation of more than 1,000 screws. Eur Spine J. 2014; 23(10): 2166-74. doi: 10.1007/s00586-014-3470-0

5. Tack P, Victor J, Gemmel P, Annemans L. 3D-printing techniques in a medical setting: a systematic literature review. Biomed Eng Online. 2016; 15(1): 115. doi: 10.1186/s12938-016-0236-4

6. Kaneyama S, Sugawara T, Sumi M, Higashiyama N, Takabatake M, Mizoi K. A novel screw guiding method with a screw guide template system for posterior C-2 fixation. J Neurosurg Spine. 2014; 21(2): 231-8. doi: 10.3171/2014.3.SPINE13730

7. Yu Z, Zhang G, Chen X, Chen X, Wu C, Lin Y, et al. Application of a novel 3D drill template for cervical pedicle screw tunnel design: a cadaveric study. Eur Spine J. 2017; 26(9): 2348-56. doi: 10.1007/s00586-017-5118-3

8. Hsieh JC, Drazin D, Firempong AO, Pashman R, Johnson JP, Kim TT. Accuracy of intraoperative computed tomography imageguided surgery in placing pedicle and pelvic screws for primary versus revision spine surgery. Neurosurg Focus. 2014; 36(3): E2. doi: 10.3171/2014.1.FOCUS13525

9. Zhang HL, Zhou DS, Jiang ZS. Analysis of accuracy of computer-assisted navigation in cervical pedicle screw installation. Orthop Surg. 2011; 3(1): 52-6. doi: 10.1111/j.1757-7861.2010.00110.x

10. John PS, James C, Antony J, Tessamma T, Ananda R, Dinesh K. A novel computer-assisted technique for pedicle screw insertion. Int J Med Robot. 2007; 3: 59-63. doi: 10.1002/rcs.117

11. Ito Y, Sugimoto Y, Tomioka M, Hasegawa Y, Nakago K, Yagata Y. Clinical accuracy of 3D fluoroscopy-assisted cervical pedicle screw insertion. J Neurosurg Spine. 2008; 9(5): 450-3. doi: 10.3171/SPI.2008.9.11.450

12. Ryken TC, Owen BD, Christensen GE, Reinhardt JM. Image-based drill templates for cervical pedicle screw placement. $J$ Neurosurg Spine. 2009; 10(1): 21-6. doi: 10.3171/2008.9.SPI08229

13. D’Urso PS, Williamson OD, Thompson RG. Biomodeling as an aid to spinal instrumentation. Spine (Phila Pa 1976). 2005; 30(24): 2841-5. doi: 10.1097/01.brs.0000190886.56895.3d

14. Van Cleynenbreugel J, Schutyser F, Goffin J, Van Brussel K, Suetens P. Image-based planning and validation of C1-C2 transarticular screw fixation using personalized drill guides. Comput Aided Surg. 2002; 7(1): 41-8. doi: 10.1002/igs.10027

15. Hu Y, Yuan ZS, Kepler CK, Albert TJ, Yuan JB, Dong WX, et al. Deviation analysis of C1-C2 transarticular screw placement assisted by a novel rapid prototyping drill template: a cadaveric study. J Spinal Disord Tech. 2014; 27(5): E181-6. doi: 10.1097/BSD.0000000000000087

16. Sugawara T, Higashiyama N, Kaneyama S, Sumi M. Accurate and simple screw insertion procedure with patient-specific screw guide templates for posterior C1-C2 fixation. Spine (Phila Pa 1976). 2017; 42(6): E340-6. doi: 10.1097/BRS.0000000000001807

17. Guo F, Dai J, Zhang J, Ma Y, Zhu G, Shen J, et al. Individualized 3D printing navigation template for pedicle screw fixation in upper cervical spine. PLoS One. 2017; 12(2): e0171509. doi: 10.1371/journal.pone.0171509

18. Wang F, Li CH, Liu ZB, Hua ZJ, He YJ, Liu J, et al. The effectiveness and safety of 3-dimensional printed composite guide plate for atlantoaxial pedicle screw: A retrospective study. Medicine (Baltimore). 2019; 98(1): e13769. doi: 10.1097/MD.0000000000013769

19. Merc M, Drstvensek I, Vogrin M, Brajlih T, Recnik G. A multi-level rapid prototyping drill guide template reduces the perforation risk of pedicle screw placement in the lumbar and sacral spine. Arch Orthop Trauma Surg. 2013; 133(7): 893-9. doi: $10.1007 / \mathrm{s} 00402-013-1755-0$ 
20. Wu ZX, Huang LY, Sang HX, Ma ZS, Wan SY, Cui G, et al. Accuracy and safety assessment of pedicle screw placement using the rapid prototyping technique in severe congenital scoliosis. J Spinal Disord Tech. 2011; 24(7): 444-50. doi: 10.1097/BSD.0b013e318201be2a

21. Lu S, Xu YQ, Chen GP, Zhang YZ, Lu D, Chen YB, et al. Efficacy and accuracy of a novel rapid prototyping drill template for cervical pedicle screw placement. Comput Aided Surg. 2011; 16(5): 240-8. doi: 10.3109/10929088.2011.605173

22. Putzier M, Strube P, Cecchinato R, Lamartina C, Hoff EK. A new navigational tool for pedicle screw placement in patients with severe scoliosis: A Pilot Study to Prove Feasibility, Accuracy, and Identify Operative Challenges. Clin Spine Surg. 2017; 30(4): E430-9. doi: 10.1097/BSD.0000000000000220 